\title{
Reversible posterior leukoencephalopathy syndrome in a patient with systemic lupus erythematosus and lupus nephritis
}

\author{
H D Samarasinghe ${ }^{1}$, K P Wanigasooriya ${ }^{1}$, S B Gunatilake ${ }^{1}$ \\ Journal of the Ceylon College of Physicians, 2012, 43, 56-57
}

\section{Background}

Reversible posterior leukoencephalopathy (RPLE) is a rapidly evolving neurologic syndrome with characteristic clinical and radiographic features. It can be one of the central nervous system presentations in systemic lupus erythematosus (SLE) though rare. Clinically, reversible posterior leukoencephalopathy syndrome is manifested characteristically by cortical blindness and visual disturbances. Neuroimaging shows bilateral subcortical and cortical edema with prominent posterior distribution. Conditions commonly associated with RPLE include hypertensive encephalopathy, eclampsia, thrombotic thrombocytopenic purpura, hemolytic uremic syndrome, immunosuppressive drugs like cyclophosphamide, cyclosporine, or methylprednisolone, and inflammatory disorders ${ }^{2,3}$. According to a study done in USA during the period from 1967 to 2005, RPLE has been identified in 13 patients with connective tissue disease. In separate case reports, 9 SLE patients, 2 Wegener's granulomatosis (WG) patients, and 1 patient with SLE and systemic sclerosis presented with RPLS ${ }^{1}$. In most cases mentioned above, complete resolution of neurological symptoms occured within 2 weeks of presentation, along with improvement or resolution of imaging abnormalities. We report here a case of RPLE in a patient with SLE.

\section{Case report}

A 17-year-old girl with SLE presented with fever, bilateral leg swelling and lethargy. On admission to the hospital she developed a generalized tonic-clonic seizure which lasted few minutes and settled with intra venous diazepam. Her blood pressure was 170/110 $\mathrm{mmHg}$. After recovery from the seizure she complained of headache and blurred vision but visual fields and bed side visual acuity tests were normal. During this admission her serum compliments (C3) were low, ANA titre was $1 / 640$, ESR $-50 \mathrm{~mm} / 1 \mathrm{st}$ hour, serum magnesium and calcium were normal, serum creatinine was $3 \mathrm{mg} / \mathrm{dl}$. Urine full report revealed proteinuria, 4-6 red cells, few pus cells and granular casts. Haemo-

\footnotetext{
${ }^{1}$ Department of Medicine, Faculty of Medical Sciences, University of Sri Jayewardenepura and Colombo South Teaching Hospital.
}

globin was $8.5 \mathrm{~g} / \mathrm{dl}$, with normocromic normocytic red cells and platelet count was $80 \times 10^{\%} / \mathrm{L}$. She was diagnosed to have an acute SLE flair up with CNS and renal involvement. Patient was started on methylprednisolone and cyclophosphamide pulse therapy since she did not respond to high dose prednisolone. She made a gradual improvement. MRI scan taken after the seizure episode showed oedema in the occipital region (Figure 1) which was suggestive of posterior leukoencephalopathy and a repeat scan two months later (Figure 2) showed complete resolution.

\section{Discussion}

When a patient with SLE develops seizures, visual disturbance and high blood pressure, possibilities are whether it is due to vasculitis or is a case of RPLE. Our patient demonstrated MRI evidence of RPLE like white matter edema and opacification in the deep occipital lobe (Figure 1) with supportive clinical evidence. As the MRI features of this condition improves in 2-3 weeks ${ }^{2,3}$ we repeated the MRI after 2 months. The repeat MRI showed complete resolution of white matter edema (Figure 2). Radiological, manifestations in RPLE is due to white matter oedema which is mainly seen in occipital, temporal and deep white matter regions. Usually complete resolutions of clinical and radiological features are seen after treatment with antihypertensives and supportive care². During this period MRI angiogram will not differentiate vasculitis from RPLE but brain biopsy which is the investigation of choice, will show differences. In SLE patients with acute neurologic symptoms like seizures associated with visual impairment, in the background of hypertension and renal impairment RPLE can be considered as a cause of their CNS presentation.

A new entity described recently ${ }^{4}$ known as reversible cerebral vasoconstriction syndrome mimics RPLE and is clinically characterised by thunderclap headache which is not typical for RPLE. While RPLE quickly progresses over a few hours, complications may occur for several days with the reversible cerebral vasoconstriction syndrome ${ }^{4}$. Cerebral angiography may show a 'string of beads' but this has also been seen in RPLE patients. Bilateral parieto-occipital lesions on MRI, typical for RPLE, are not characteristic for the reversible cerebral vasoconstriction syndrome. Nevertheless, in about $10 \%$ of cases there seems to be overlap between this syndrome and RPLE. 


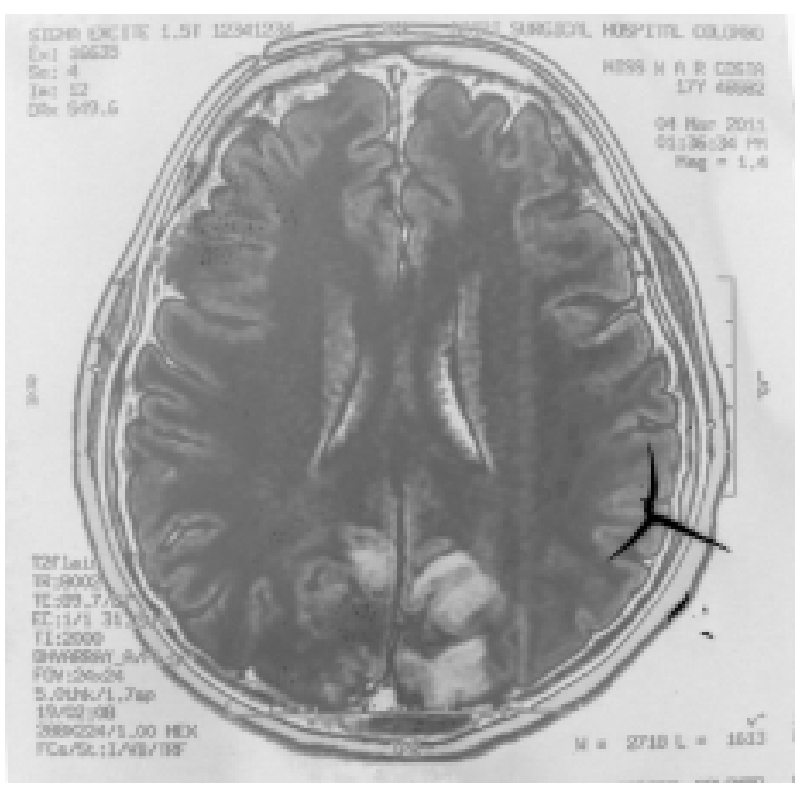

Figure 1.

\section{Clinical points}

- RPLE is a clinicoradiological syndrome and its symptoms and signs develop over hours - a combination of seizures, disturbed vision, altered mental function and headache.

- MRI is the diagnostic gold standard. The predominant affected region is parieto-occipital of both hemispheres. The subcortical white matter is always affected but with frequent involvement of the cortex also. Typical RPLE lesions on MRI are thought to represent vasogenic oedema.

- Both children and older people can be affected without any gender differences.

- There are many different trigger factors, most commonly abrupt hypertension, renal failure, immunosuppressive therapy, eclampsia, autoimmune disease and infections.

- The prognosis is good and recurrence rare.

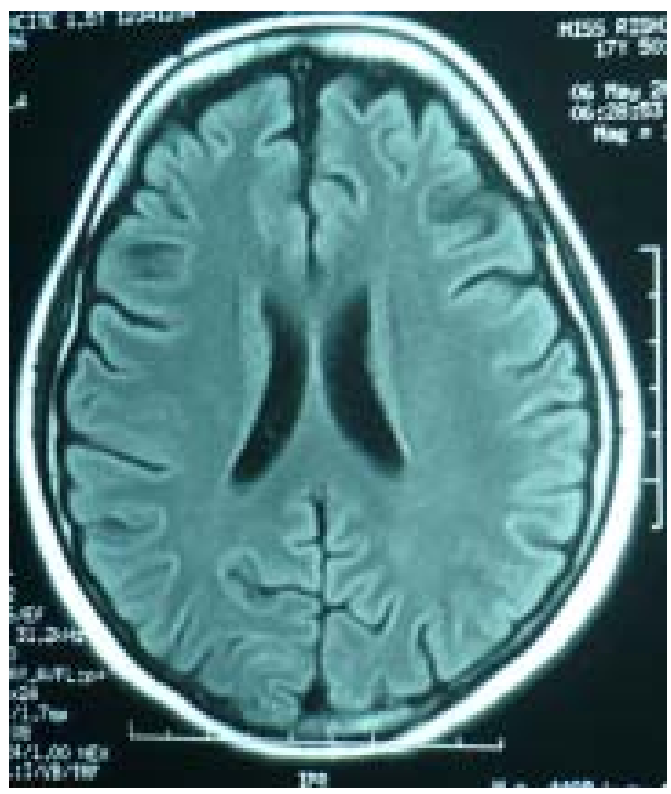

Figure 2.

Symptoms generally resolve within a week. MRI lesions resolve somewhat more slowly.

- Treatment consists of antihypertensive drugs, withdrawal of medication such as chemotherapy, and treatment of any underlying disease.

\section{References}

1. Min L, Zwerling J, Ocava LC, Chen IH, Putterman C. Reversible posterior leukoencephalopathy in connective tissue diseases. Semin Arthritis Rheum 2006; 35(6): 388-95.

2. Magnano MD, Bush TM, Herrera I, Altman RD. Reversible posterior leukoencephalopathy in patients with systemic lupus erythematosus. Semin Arthritis Rheum 2006; 35(6): 396-402.

3. Roth C, Ferbert A. The posterior reversible encephalopathy syndrome: what's certain, what's new? Pract Neurol 2011; 11: $36-144$.

4. Ducros A, Bousser MG. Reversible cerebral vasoconstriction syndrome. Pract Neurol 2009; 9: 256-67. 